\title{
Influence of pore-size distributions and pore-wall mechanics on the mechanical behavior of cellular solids like aerogels
}

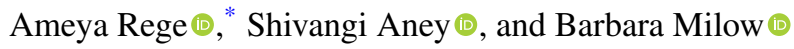 \\ Department of Aerogels and Aerogel Composites, Institute of Materials Research, German Aerospace Center, \\ Linder Höhe, 51147 Cologne, Germany
}

(Received 10 November 2020; accepted 18 March 2021; published 2 April 2021)

\begin{abstract}
The pore-size distributions play a critical role in the determination of the properties of nanoporous cellular materials like aerogels. In this paper, we propose a micromechanical model, and by further designing artificial normal pore-size distributions, we inspect their effect on the macroscopic stress-strain curves. We show that the location of the mean pore size as well as the broadness of the distribution strongly affects the overall macroscopic behavior. Moreover, we also show that by using different damage criteria within the proposed model, the elastic, inelastic, and brittle nature of the macroscopic material can be captured. The damage criteria are based on the different modes of deformation in the pore walls, namely, elastic buckling, irreversible bending and brittle collapse under compression, and combined bending and stretching under tension. The proposed model approach serves as a reverse engineering tool to develop cellular solids with desired mechanical properties.
\end{abstract}

DOI: 10.1103/PhysRevE.103.043001

\section{INTRODUCTION}

Open-porous cellular solids are characterized by a complex mechanical behavior. Depending on the properties of their skeletal (or solid) material, they show either an elastic, elasto-plastic, or brittle response. Diverse attempts [1-7] have been made to model and characterize the mechanical behavior of such cellular materials within a micromechanical or phenomenological framework. To that end, the most extensive work has been accomplished by Gibson and Ashby [2]. They showed that a simple approach of modeling the mechanisms of deformation and failure by means of a two-dimensional (2D) system without specifying the exact cell geometry proves to be accurate and useful in predicting the mechanical properties of such open-porous cellular solids. Under compression, such cellular solids generally show three distinct regimes: (1) a linear elastic regime, where local bending and buckling of the pore walls occur, (2) a plateau regime, where pore collapse takes place, and (3) a densification regime, where the hardening due to compaction of the collapsed pores occurs. While cellular solids exhibit complex phenomena, their mechanical properties can be well established based on their cell wall mechanics. The open-cell foam model by Gibson and Ashby [2] is widely used for estimating several mechanical properties, such as the Young's modulus and yield stress, of cellular materials. Such materials maximize their mechanical properties with increasing density. The well-known relation between the Young's modulus $(E)$ and density $(\rho)$,

$$
E \propto \rho^{m},
$$

where $m$ is the scaling exponent, is a good example. Based on the open-cell foam model by Gibson and Ashby, $m=2$.

*ameya.rege@dlr.de
However, for cellular materials that rather show a random network connectivity, the exponent may vary between 1 and 4. This is generally the case for aerogels and similar materials $[8,9]$.

In the past decade, there has been a strong interest in the development of nanoporous open-cellular materials given their exceptional thermal insulation properties. Having pore sizes in the Knudsen regime, the resulting materials show exceptionally low thermal conductivities [10]. While heat flow is a scalar problem being a function of the temperature gradient, the mechanical deformation is a vectorial one [11]. Moreover, maximizing the mechanical stiffness of the cellular solid while maintaining a low thermal conductivity results in a conflict of interest, given that both properties are essentially functions of the solid phase fraction of the porous cellular material. To this end, analyzing the nanoporous microstructure and its influence on the mechanical properties is important for designing materials for specific applications. Many nanoporous solids, such as biopolymer aerogels and specifically cellulose ones, show an exponent $m \approx 2$ given their foamlike appearance [12]. The mechanical properties of these materials are highly controlled by their pore structure [7]. This is typically quantified by the pore-size distribution. There has been recent interest in the characteristics of the pore sizes and pore-size distributions [13-16], as well as their effect on the properties of the materials [17-20]. Many nanocellular solids such as biopolymer aerogels exhibit pore sizes ranging from a very few nanometers up to 100$150 \mathrm{~nm}$. Recently at the International Seminar on Aerogels 2020, questions pertaining to the effect of the pore size and more specifically pore-size distributions on the macroscopic mechanical behavior of aerogels were raised. Answering this question from an experimental perspective is rather challenging. This is because it is extremely difficult to only tune the pore size and distribution alone, while keeping all other 
(d) Modes of cell wall/

(a) Reference configuration configuration strut deformation

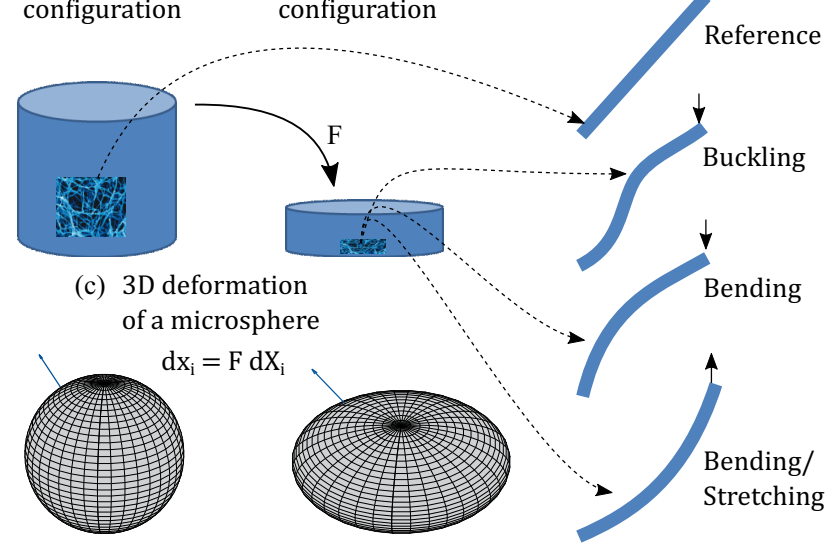

FIG. 1. An illustration of a macroscopic material specimen (a) in an undeformed configuration and (b) in a deformed configuration upon compression, (c) deformation of a microsphere subject to a compressive deformation gradient $\mathbf{F}$, and (d) different modes of compressive or tensile deformation of the cell walls or struts: buckling, bending, and bending and stretching. The inset showing cellular microstructure in (a) and (b) is that of cellulose aerogels reproduced from Ref. [6] with permission from The Royal Society of Chemistry.

parameters constant, while synthesizing a porous material such as an aerogel. Accordingly, a theoretical approach would be better suited to give an explanation. In our previous work [21], we have proposed a simple physically based constitutive model that described the macroscopic mechanical behavior of the cellular solid based on their pore-wall mechanics where the pore-size distribution was considered. There a special case of uniaxial tension for a brittle cellular material was presented.

In this paper, a generalized framework for numerical modeling of open-porous cellular solids is presented. Here a focus on the influence of pore-size distributions, in particular, the location of the mean of a normal pore-size distribution as well as the broadness of the peak, on the macroscopic mechanical behavior of nanocellular solids is described. The effect of the range of the pore-size distribution is also accounted for. Furthermore, the effect of the choice of the damage criteria on modeling the constitutive behavior of cellular solids is investigated by generalizing our previously published model. The cell walls of a cellular solid may undergo elastic buckling in the case of a flexible solid or irreversible bending in the case of an elasto-plastic solid or sudden failure beyond the elastic regime in the case of brittle solids [3]. Under tension, the walls first try to align themselves to the direction of loading and then undergo stretching [22]. During the aligning process, bending is more dominant, followed by stretching. These modes are exemplarily shown in Fig. 1. Figures 1(a) and 1(b) show a macroscopic cellular solid under compressive deformation, while Fig. 1(d) shows the above-mentioned modes of deformation. To the best of our knowledge, very few studies have reported the modeling of the macroscopic stress-strain response of cellular solids by accounting for the different modes of damage within a numerical micromechanical framework.
The paper is divided as follows. In Sec. II the micromechanical model is proposed. The results of the model are illustrated, and an explanation is presented in Sec. III. There, first, the effect of the pore sizes and distributions on the macroscopic constitutive behavior are illustrated and analyzed. Then the effect of different damage mechanisms on the overall stress-strain nature and the failure of the network are described. Last, the conclusions from this study are drawn in Sec. IV.

\section{MODEL}

For any given open-porous cellular material, the macroscopic mechanical behavior is governed by the mechanism of deformation of their micro- (or nano-)scopic pore walls. In our previous works [7,21], micromechanical models were presented based on the assumption that the material network is made up of idealized microcells having an isotropic spatial distribution. An idealized microcell is referred to as a square-shaped cell, representing a 2D pore. Special cases of damage were considered based on the type of material being considered. In this section, a generalized approach to modeling open-porous materials is proposed, based on our previous works. The one-dimensional (1D) network strain energy of an idealized microcell is expressed as

$$
\stackrel{\boldsymbol{d}}{\Psi}=\int_{l_{\text {active }}} N_{0} p(l)\left(\psi_{\mathrm{bn}}+\psi_{\mathrm{st}}\right) d l,
$$

where the bending energy $\psi_{\text {bn }}$ and the stretching energy $\psi_{\text {st }}$ based on the deformation of the pore walls are given as

$$
\begin{aligned}
& \psi_{\mathrm{bn}}=\int_{0}^{l} \frac{1}{2} \kappa\left(\phi^{\prime}\right)^{2} d l, \\
& \psi_{\mathrm{st}}=\int_{0}^{l} \frac{1}{2} \mu\left(u^{\prime}\right)^{2} d l,
\end{aligned}
$$

where $\phi^{\prime}$ is the curvature and $u^{\prime}$ is the axial strain along the pore walls where the pore-wall length ranges between $l_{\min }$ and $l_{\max } . \kappa$ and $\mu$ represent the bending and stretching stiffness, respectively. The parameters $l$ and $l_{\text {active }}$ denote the length of the given pore wall and the range of active (not collapsed or failed) microcells in the network, respectively. $l_{\text {active }}$ will be addressed in more detail in the following. $p(l)$ is the pore-size distribution of the given material. For most nanocellular porous solids, such as aerogels, the pore sizes are far from being constant. These pore sizes, for mesoporous aerogels, are experimentally estimated by means of the Barrett-Joyner-Halenda (BJH) model [23], which is applied to the data obtained from nitrogen sorption isotherms. $N_{0}$ is the total number of cells within the network. This parameter is a function of the relative density.

The total strain energy of the three-dimensional (3D) network is then obtained as the sum of the energies in the different spatial directions. Applying an isotropic spatial distribution, i.e., idealized cells (pore walls) are spread equally in all directions, one can express

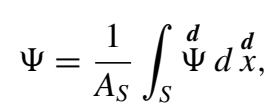




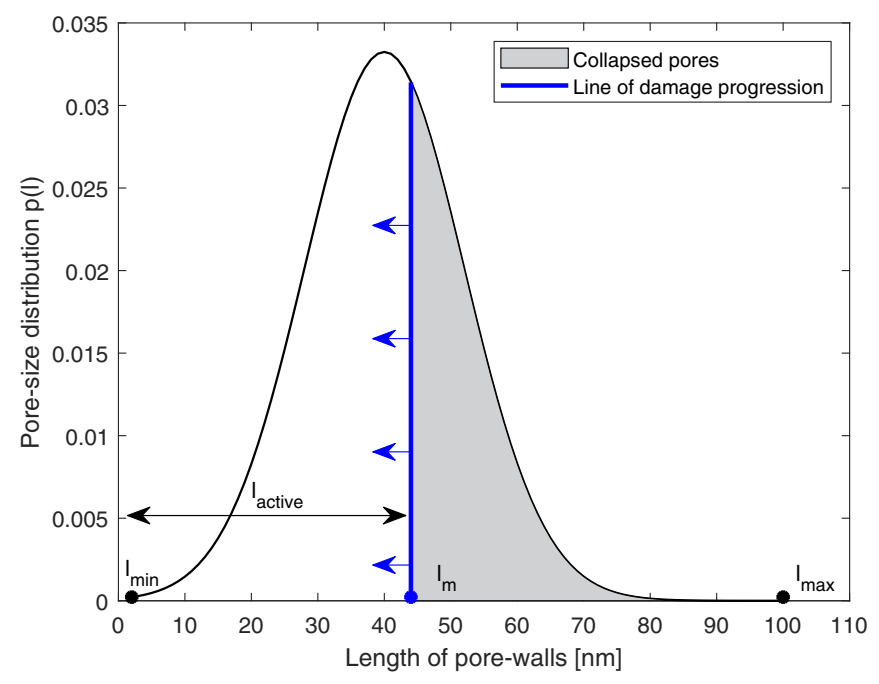

FIG. 2. Illustration of the damage evolution in a cellular network based on collapse of pores by buckling. The blue colored arrows indicate the damage evolution.

where $A_{S}$ represents the surface area of the unit sphere. For the purpose of generalization from a $1 \mathrm{D}$ response to a $3 \mathrm{D}$ one, directional averaging by means of the numerical integration over the unit sphere [24] was applied. The integration in Eq. (4) was accordingly carried out numerically as

$$
\Psi \approx \sum_{i=1}^{k} \omega_{i}^{\stackrel{d_{i}}{\Psi}},
$$

where $\omega_{i}$ are weight factors corresponding to the collocation directions $\boldsymbol{d}_{i}(i=1,2,3, \ldots, k)$. This method was recently shown to be effective for $3 \mathrm{D}$ generalization in the case of modeling porous materials $[7,25]$.

Under compression, three different modes of damage were considered. These were (1) pore collapse due to elastic buckling of the pore walls, (2) pore collapse due to irreversible bending of the pore walls, and (3) pore collapse due to brittle failure of the pore walls. The Euler buckling criterion was used to model case (1). This means that once the critical buckling load in a pore wall was reached, the pore was considered to collapse. This changed the range of $l_{\text {active }}$. For example, on the basis of the buckling criterion, the larger pores collapsed first (see Fig. 2), because the slenderer pore walls buckled sooner than the shorter ones. Accordingly, the value of $l_{\max }$ gets updated to a new value $l_{m}$. Note that, in the reference state, $l_{m}=l_{\max }$, and then $l_{\min }<l_{m}<l_{\max }$. To model case (2), the bending moment criterion was used. Here the pore walls were considered to undergo irreversible deformation after the bending moment of the wall $M_{w}$ reached its maximal allowable value. Beyond this point, where the yield stress in the pore wall is reached, a plastic hinge would appear [2]. Last, to model case (3), the normal stress criterion was applied. In this case, a combined bending and compression were considered to result in the failure of the pore walls. Under tension, the damage was again based on the normal stress criterion, as the pore walls would first try to align themselves to the direction of loading (a bending-dominant behavior) and then begin to stretch with increasing deformation.
In the following, first the effect of the pore sizes and poresize distribution is analyzed. This is followed by exploring the application of the different above-mentioned damage criteria and their effect on the overall macroscopic stress-strain curves.

\section{RESULTS}

\section{A. Effect of pore sizes and pore-size distributions}

To account for the effect of pore sizes, two sets of examples were chosen. A standard Gaussian distribution was chosen to describe the pore-size distribution over a range between 2 and $100 \mathrm{~nm}$. This encompasses the mesopores, those between 2 and $50 \mathrm{~nm}$, and macropores, those larger than $50 \mathrm{~nm}$, according to the IUPAC definition. Realistic pore sizes in materials like aerogels are usually non-Gaussian and can be approximated by, e.g., a generalized $\beta$ distribution function [26]. In this work, for the purposes of analyzing the effect of pore sizes in an open-porous cellular material, a normal distribution was considered. First, the mean pore size was considered to vary in different examples, from 20 to $80 \mathrm{~nm}$, as illustrated in Fig. 3(a). For all other model parameters kept constant, purely varying the mean pore size had a very strong effect on the macroscopic tensile and compressive behavior. Figure 3(b) illustrates this effect on the tensile stress-strain curve. It shows that as the mean pore size was shifted to the right, meaning it became larger, the constitutive behavior softened. However, it solely did not seem to have a very strong impact on the brittle failure of the network. Here an important point has to be considered. For any given network with a constant number of cells and having a constant pore-wall thickness, if the poresize distribution is changed, the relative density is bound to be affected. This can be controlled by adjusting other parameters, such as the pore-wall thickness. Conventional foams, for, e.g., metal foams, do not show pore-size dependency over the mechanical properties [27]. However, such foams also do not boast pore sizes with such variations, from 2 to $150 \mathrm{~nm}$. In our model, since the relative density itself does not appear to be a parameter, keeping it constant is not necessarily possible. Hence, the above-illustrated observations may appear to be an effect of the corresponding change in the relative density. However, recent observations in carbon aerogels [28,29] have shown that aerogels prepared with the same relative density, but with varying pore sizes, show different mechanical properties. Carbon aerogels with smaller pores behave more stiffly than those with larger pores, although both have similar relative densities. This was precisely demonstrated by the results shown in Figs. 3(a) and 3(b). Further investigations appear necessary to explore such size effects, specifically in combination with the relative density. Simultaneous changes to the pore-wall thickness may certainly alter the above-stated conclusion. The larger the pore-wall thickness, the stiffer is the material response. Under compression, the response also showed a similar softening. For example, when the mean pore size was changed from 20 to $80 \mathrm{~nm}$, the mechanical stiffness dropped by a factor of over one-third. The blue curve in Fig. 3(e) shows the softening of the Young's modulus as measured from the uniaxial compression simulations. The softening of the mechanical stiffness was rather steep, 

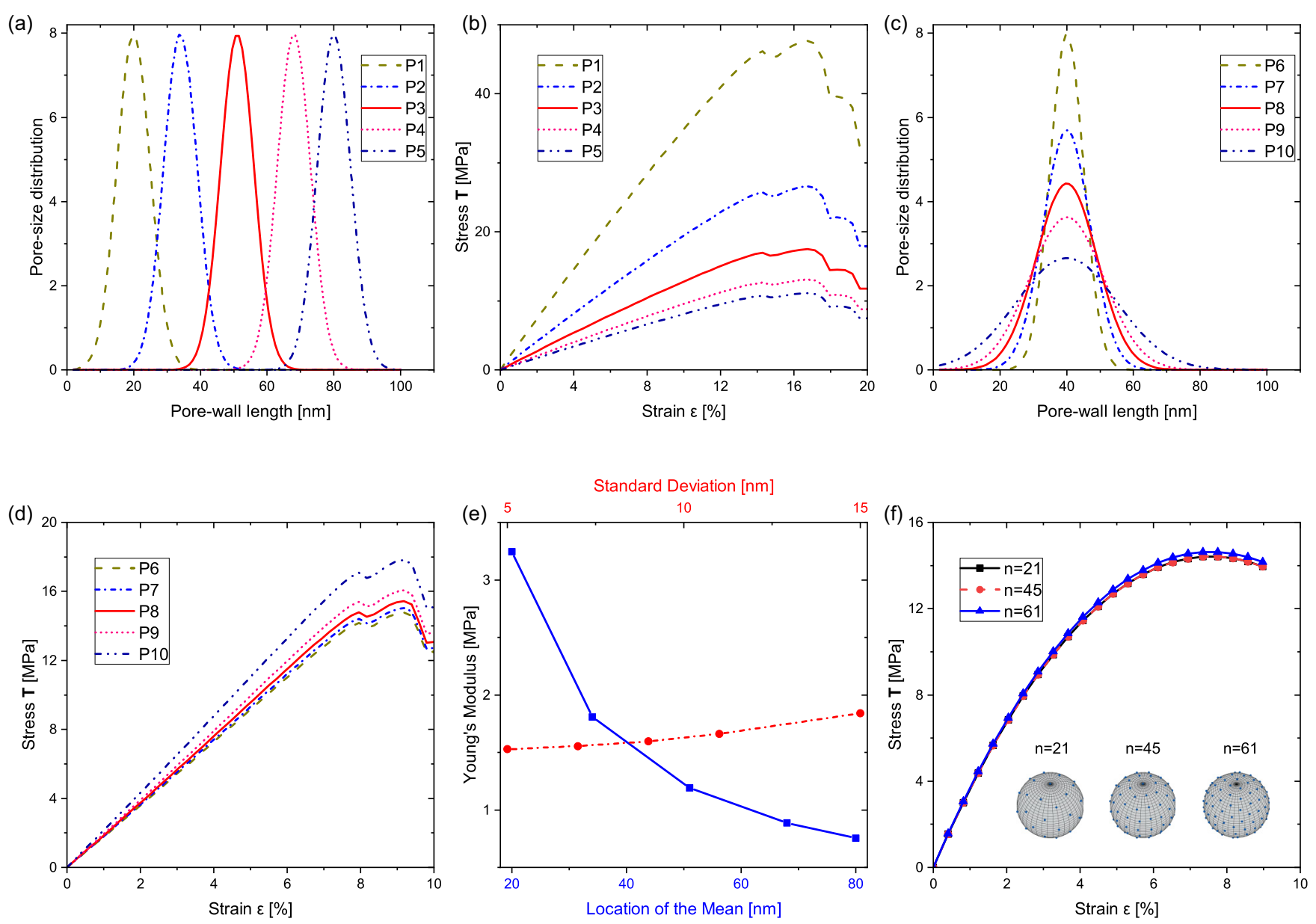

FIG. 3. (a) The different pore-size distributions considered, based on the variation of the mean pore sizes, (b) the influence of the mean pore size on the macroscopic tensile behavior, (c) the different pore-size distributions considered, based on the variation of the standard deviation in the pore sizes, (d) the influence of the standard deviation on the macroscopic tensile behavior, (e) the Young's modulus vs the mean and standard deviation of the pore-size distribution, and (f) the effect of the choice of numerical scheme within the microsphere model on the stress-strain behavior (inset shows the three schemes, $n=21,45$, and 61).

although it seemed to be flattening with every increase in the mean value of the pore sizes. The conclusions drawn from Figs. 3(a), 3(b), and 3(e) corroborate well observations from experiments as elucidated above. However, for a general application of the model, care must be taken to analyze this effect in correlation with the changes in the relative density.

It was of further interest to investigate the effect of the broadness of the peak on the overall mechanical behavior. Thus, the standard deviation in the considered normal distribution was varied. As seen from the pore-size distributions of various aerogels [26], not all have a very strong peak, but some have a rather flattened one. Figure 3(c) shows the different pore-size distributions modeled purely varying the standard deviation. For a given mean, the results are not very dramatic. Figure 3(d) shows the tensile stress-strain behavior for the corresponding different pore-size distributions. The wider the standard deviation, slightly stiffer is the mechanical response. This is more evident from Fig. 3(e), where the Young's modulus, as measured under compression, showed a minor increase with widened standard deviation. This conclusion may certainly change if other parameters are simultaneously altered. Thus, for obtaining the significant influence on the macroscopic behavior, it is more important to reverse engineer the location of the mean of the poresize distributions in nanoporous cellular materials. While the above-mentioned conclusions remain valid also for a few non-Gaussian pore-size distributions, e.g., the generalized $\beta$ distribution function that adheres to the distributions that are observed in polysaccharide aerogels, they may need further attention when modeling open-porous networks with other different types of pore-size distributions that do not exhibit Gaussian nature.

It was also necessary to investigate the effect of the choice of the directional scheme on the overall model response. Numerical integration over a unit microsphere is predominantly used for directional averaging in the modeling of the network response of elastomers [30]. Recently this approach has been applied for cellular solids $[7,25]$. The compression of an exemplary microsphere subjected to a deformation gradient F is illustrated in Fig. 1(c). Figure 3(f) shows the effect of using different numerical schemes in the presented model on the macroscopic stress-strain behavior. All the responses coalesced, suggesting that the model is insensitive to the direction scheme applied. In our model, we have used the scheme of 

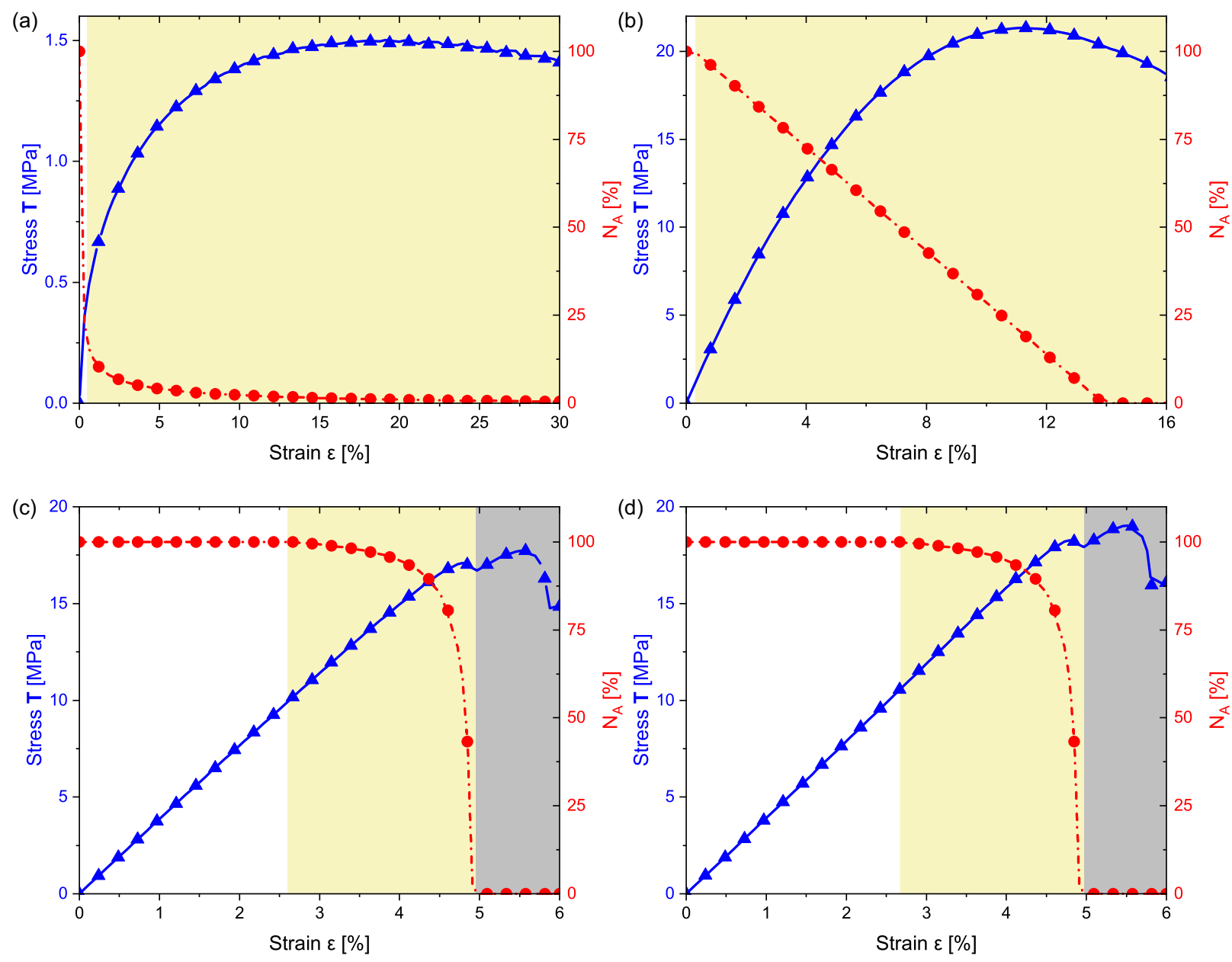

FIG. 4. Effect of different damage mechanism criteria, (a) elastic buckling, (b) irreversible bending, and (c) brittle collapse, on the compressive response of the cellular solids. (d) The same is illustrated for the failure due to combined bending and stretching under tension showing brittle failure. The shading color scheme is as follows: the white region shows the linear-elastic regime, yellow the pore collapse, and gray the material failure. The line type scheme is as follows: the blue solid line with triangle points shows the stress-strain curve, and the red dotted line with circle points illustrates the collapse of pores.

45 integration points over the half sphere, as it was shown to provide the best results by considering a trade-off between computational time and numerical accuracy [31].

\section{B. Effect of different damage criteria}

The different deformation modes for cellular solids under tension or compression have been very well outlined by Gibson and Ashby [2]. In the majority of the material models based on the pore-wall mechanics, the relationship in Eq. (1) is explored, but the macroscopic mechanical behavior of the cellular solid based on the microstructural parameters is not simulated. Finite element methods or other similar ones are applied for this purpose. However, the work of Gibson and Ashby forms a very strong basis for defining a numerical model. As mentioned above, they showed that a simple approach of modeling the mechanisms of deformation and failure by means of a $2 \mathrm{D}$ cellular system without specifying the exact cell geometry proves to be accurate and useful in predicting the mechanical properties of cellular solids. In this paper, different damage criteria have been introduced and subsequently varied within the framework of the proposed model for describing diverse macroscopic stress-strain curve types of cellular networks.

Considering compression, Gibson [3] outlined elastic buckling as the first mode of deformation. Accordingly, by modeling Euler buckling in the pore walls under compression, the macroscopic stress-strain curve was modeled as shown in Fig. 4(a). The pore collapse begins with the onset of critical buckling stress in the respective pore walls of the material. This is quantified by the factor $N_{A}$ in the plot, which corresponds to the active cells that are oriented in the direction of loading. Thus, in the presented case, the elastic buckling was seen to occur even at very small strains. For a very soft material, the walls would buckle even at very small strains, thus expediting the pore collapse. The white region in Fig. 4(a) shows the linear elastic region, whereas the yellow-shaded region begins with the onset of the first buckling of pore walls in the network. The range of the linear elastic (white) region varies upon the geometric parameters as well as the Young's modulus of the pore walls. This is in full agreement with the Gibson and Ashby open-cell foam model. The directional dependency of the walls oriented in the other directions was accounted for by the directional averaging. The mechanism 
for densification was not accounted for, hence the stress-strain curve shows only the linear elastic regime followed by the softening effect due to the pore collapse. This means, while integrating over the pore-size distribution, only the active pore walls or struts were accounted for $\left(l_{\text {active }}\right.$; see Fig. 2$)$. If the Young's modulus of the pore walls, their thickness, and the pore-size distribution is known beforehand, the macroscopic mechanical stress-strain curve can be simulated.

The second mode of deformation was irreversible bending. Figure 4(b) shows the macroscopic compressive response of a cellular solid modeled with the same microstructural parameters, but changing only the damage criterion. Here the pore walls were considered to undergo irreversible deformation after the bending moment of the pore wall reached its maximal allowable value. Here, also, the model was able to predict the linear elastic regime followed by the softening due to pore collapse. Again, the range of the linear elastic regime was strongly affected by the Young's modulus and geometric parameters of the pore walls. The observed collapse of pores was relatively gradual in comparison to the one observed in Fig. 4(a) and followed the local bending of the walls in the network.

The third mode of deformation was brittle failure. Figure 4(c) shows the response of a cellular solid subject to compression and subsequent brittle failure. Here the gray region represents network failure. In this case, the collapse of the pores was modeled based on the normal stress criterion. Once the normal stress in the pore walls reached the maximal allowable value, the pores were considered to collapse. One can distinctly observe the linear elastic regime, followed by the onset of damage, which propagates very rapidly as expected for a brittle material, leading to macroscopic failure. Such brittle failure is clearly seen in brittle nanoporous materials under compression, such as carbon aerogels [29]. Figure 4(d) shows the stress-strain curve modeled under tension. In this case, the pore walls try to align themselves to the direction of loading. This results in a combined local bending and stretching of the walls followed by a more stretching dominated one [22]. As can be visualized in Fig. 4(d), $N_{A}$ again showed that the microcells oriented in the direction of loading begin to fail on a microscopic level leading to macroscopic network failure. Thus, the model captured the brittle failure under compression as well as under tension. It is observed that, for a given set of parameters, applying the failure criterion due to normal stress, under tension and compression, resulted in similar-appearing stress-strain curves.

The results presented in Fig. 4 show that by choosing an appropriate damage criterion within the framework of the proposed model, the macroscopic stress-strain response can be effectively described. In conventional foams, it has been observed that beyond a critical limit pertaining to size effects, the network response should be independent of the number of cells in the network. Since our model is based on the network strain energy, the parameter appears as purely a multiplicative factor but was nonetheless crucial in analyzing the progression of damage in the cellular network. This made predicting the network failure possible. A current limitation to the model arises from its inability to capture the densification of the network within the presented micromechanical framework. In the current model, the strain energy is calculated only over the active pore walls or struts $\left(l_{\text {active }}\right)$. However, the collapsed pore walls play a significant role in contributing towards the compaction of the network and need to be accounted. Moreover, the current model assumed affine deformation. This means that the microscopic stretches in the pore walls or struts followed the macroscopic deformation gradient. There have been alternative approaches for accounting the nonaffine deformation in the literature [22] and shall be considered in our next work.

\section{CONCLUSIONS}

A micromechanical constitutive model to predict the macroscopic stress-strain curves of nanoporous open-cellular solids, based on the mechanics of the pore walls or struts, is proposed in this paper. The following conclusions can be deduced from the presented work. The nature of pore sizes and their distributions strongly affect the mechanical properties. By considering a normal distribution function for mimicking the pore-size distribution in a cellular solid, it is realized that tailoring the location of the mean pore size is significantly more important than controlling only the standard deviation while maintaining a constant mean pore size. With increasing pore size, and for all other parameters constant, the network response softens. As long as the mean pore size is constant, adjusting the range of the pore sizes shows no significant effect on the mechanical properties. These conclusions might need minor adjustments while dealing with non-Gaussian pore-size distributions. Thus, the proposed model presents an alternative approach of modeling open-porous cellular solids, by accounting for the pore-size distributions.

It further accounts for the different modes of damage, namely, elastic buckling, irreversible bending, and brittle failure. Thus, the model is capable of predicting elastic and inelastic effects in cellular solids. For the case that all model parameters-pore-wall thickness, Young's modulus of the pore wall, and the pore-size distribution of the considered open-porous cellular material-are known a priori, the macroscopic tensile and compressive stress-strain curves can be predicted. Such a modeling approach could be effectively used to reverse engineer the properties of nanoporous cellular materials like aerogels, by first correlating the synthesis parameters to the model ones, and then accordingly adjusting the synthesis parameters.

\section{ACKNOWLEDGMENT}

This work was supported by the Next Generation Car project at the German Aerospace Center. S.A. acknowledges the DLR-DAAD Research Fellowship program.
[1] C. M. Ford and L. J. Gibson, Int. J. Mech. Sci. 40, 521 (1998).

[2] L. J. Gibson and M. F. Ashby, Cellular Solids: Structure and Properties (Cambridge University Press, Cambridge, 1999).
[3] L. J. Gibson, J. Biomech. 38, 377 (2005).

[4] S. Diebels, T. Ebinger, and H. Steeb, J. Mater. Sci. 40, 5919 (2005). 
[5] C. Jo, J. Fu, and H. E. Naguib, Polymer 46, 11896 (2005).

[6] M. I. El Ghezal, Y. Maalej, and I. Doghri, Comput. Mater. Sci. 70, 51 (2013).

[7] A. Rege, M. Schestakow, I. Karadagli, L. Ratke, and M. Itskov, Soft Matter 12, 7079 (2016).

[8] J. Groß and J. Fricke, Nanostruct. Mater. 6, 905 (1995).

[9] Z. Qin, G. S. Jung, M. J. Kang, and M. J. Buehler, Sci. Adv. 3, e1601536 (2017).

[10] L. W. Hrubesh and R. W. Pekala, J. Mater. Res. 9, 731 (1994).

[11] S. Torquato, Random Heterogeneous Materials: Microstructure and Macroscopic Properties (Springer-Verlag, New York, 2002).

[12] S. Zhao, W. J. Malfait, N. Guerrero-Alburquerque, M. M. Koebel, and G. Nyström, Angew. Chem. Int. Ed. 57, 7850 (2018).

[13] S. A. Hertel, X. Wang, P. Hosking, M. C. Simpson, M. Hunter, and P. Galvosas, Phys. Rev. E 92, 012808 (2015).

[14] V. Langlois, V. H. Trinh, C. Lusso, C. Perrot, X. Chateau, Y. Khidas, and O. Pitois, Phys. Rev. E 97, 053111 (2018).

[15] D. Lee, J. Kim, S. Kim, G. Kim, J. Roh, S. Lee, and H. Han, Microp. Mesop. Mater. 288, 109546 (2019).

[16] S. Song, Q. Ding, and J. Wei, Phys. Rev. E 100, 053314 (2019).

[17] T. A. Kuder and F. B. Laun, Phys. Rev. E 92, 022706 (2015).

[18] S. Bakhshian and M. Sahimi, Phys. Rev. E 94, 042903 (2016).

[19] S. F. Plappert, J.-M. Nedelec, H. Rennhofer, H. C. Lichtenegger, and F. W. Liebner, Chem. Mater. 29, 6630 (2017).
[20] D. Hou, D. Li, P. Hua, J. Jiang, and G. Zhang, Cem. Concr. Compos. 96, 11 (2019).

[21] A. Rege and M. Itskov, Acta Mech. 229, 585 (2018).

[22] P. R. Onck, T. Koeman, T. van Dillen, and E. van der Giessen, Phys. Rev. Lett. 95, 178102 (2005).

[23] E. P. Barrett, L. G. Joyner, and P. P. Halenda, J. Am. Chem. Soc. 73, 373 (1951).

[24] Z. P. Bažant and B. H. Oh, Z. Angew. Math. Mech. 66, 37 (1986).

[25] T. Bleistein, A. Jung, and S. Diebels, Contin. Mech. Thermodyn. 32, 255 (2019).

[26] A. Rege, I. Preibisch, M. Schestakow, K. Ganesan, P. Gurikov, B. Milow, I. Smirnova, and M. Itskov, Materials 11, 1670 (2018).

[27] W.-Y. Jang and S. Kyriakides, Int. J. Solids Struct. 46, 635 (2009).

[28] M. Schwan, Synthese und Eigenschaften flexibler ResorcinFormaldehyd- und Kohlenstoffaerogele, Ph.D. thesis, Rheinisch-Westfälischen Technischen Hochschule Aachen (2018).

[29] A. Rege, M. Schwan, L. Chernova, M. Hillgärtner, M. Itskov, and B. Milow, J. Non-Cryst. Solids 529, 119568 (2020).

[30] R. Dargazany and M. Itskov, Phys. Rev. E 88, 012602 (2013).

[31] A. E. Ehret, M. Itskov, and H. Schmid, Int. J. Numer. Methods Eng. 81, 189 (2010). 